\title{
Determination of sulfonamide antibiotics in sludge by ultrasonic solid phase extraction and HPLC
}

\author{
Xiaoxia Cao ${ }^{a}$, Dengxin $\mathrm{Li}^{\mathrm{b}}$ \\ ${ }^{1}$ School of Environmental Science \& Engineering, Donghua University, Shanghai 201620, China \\ acaoxiaoxiadarrouy@163.com, blidengxin@dhu.edu.cn
}

\begin{abstract}
Keywords: Ultrasonic solid phase extraction -HPLC; Sulfonamide antibiotics; High performance liquid chromatography (HPLC); Sludge

Abstract: In order to improve the recovery rate of sulfonamide antibiotics in sludge, and to optimize the extraction and determination methods of sulfonamides antibiotics in sludge, ultrasonic solid phase extraction -HPLC was used for the detection of sulfonamides antibiotics in sludge. First the determination of the test factors by single factor test was conducted, then the extraction conditions were optimized by orthogonal experiment. When the extraction ratio of $\mathrm{V}$ (acetonitrile): $\mathrm{V}$ (water) was $=1$ : 3, oscillation time was $20 \mathrm{~min}$, ultrasonic frequency was $35 \mathrm{~Hz}$, ultrasonic time was $15 \mathrm{~min}$, and extraction agent was $20 \mathrm{ml}$, when the average content of extraction of sulfonamide antibiotics reached maximum, the recovery rates of Sulfadiazine, and sulfamethazine antibiotics were: $75.50 \%$ and $72.79 \%$ respectively. This indicates that the detection method can determine the content of sulfonamide antibiotics in the sludge effectively.
\end{abstract}

\section{Introduction}

In recent years, the environmental researchers pay more attention to the harmful and toxic effects antibiotic medicines in wastewater as a new type of pollutants in the environment. ${ }^{[1]}$ Antibiotics are mainly used in the prevention and treatment of bacterial infections, and is one of the most widely used drugs in the world. Antibiotics have a potential impact on the development and spread of antimicrobial resistance. At present, the problem of antibiotic contamination has been listed as an important environmental problem in many developed countries in the first 20 years of twenty-first Century, and the lots of research has been going on to contain it ${ }^{[2]}$. It has been reported that in 2003, the output of sulfonamides antibiotics in China was more than $20000 \mathrm{t}^{[3]}$, which is widely used in clinical, animal agriculture and aquaculture ${ }^{[4]}$. Antibiotics, as human pharmaceuticals, are digested and excreted into the environment by human metabolites and are in unaltered form. At present, the antibiotic residues in drinking water ${ }^{[5]}$, domestic sewage, soil, river sediment and sludge ${ }^{[6-7]}$ and other environment has been detected. As the sewage treatment plant is considered to be the hub of various pollutants, the part of the drug is lipophilic that can enrich easily in sludge system, therefore, the detection of antibiotics concentration in the sludge can be as high as to be measured in $\mathrm{mg} / \mathrm{kg}$, which seriously is affecting the sludge compost, resource use, harmless disposal of sludge disposal and subsequent processing. In order to understand the existing level and pollution status of antibiotics in sludge, it is necessary to establish an analytical method for the determination of antibiotics in sludge, so as to test the actual samples.

Due to the complexity of the sludge matrix, there are many kinds of target drugs having different physical and chemical properties. In order to reduce the interference and improve the extraction efficiency, the samples extraction and purification steps are critical. Mingyue Li et al. ${ }^{[8]}$ used matrix solid-phase dispersion and high performance liquid chromatography tandem mass spectrometry to analyze the typical antibiotics in sludge, and the recovery rate of sulfonamides was $40.15 \%-63.75 \%$; Shuo Wang et al. ${ }^{[9]}$ used ultrasonic extraction and high performance liquid chromatography tandem mass spectrometry to determine the antibiotic in sludge, and the recovery rate was $46.1 \%-69.6 \%$; Xiaoxia Jiang ${ }^{[10]}$ used ultrasonic extraction, HLB column pre-concentration and ultra-performance liquid chromatography mass spectrometry, with $10 \mathrm{ml} 0.2 \mathrm{M}$ citric acid buffer and $10 \mathrm{ml}$ acetonitrile as an extraction agent, to determine the antibiotic in sludge sediment, and the recovery rate was 61.2\%-96.3\%; Jiali Ding et al. ${ }^{[11]}$ used ultrasonic extraction, SAX-HLB column enrichment and HPLC tandem mass spectrometry, with methanol, $0.1 \mathrm{~mol} / \mathrm{L}$ EDTA-2Na solution, and citric acid 
buffer $(\mathrm{pH}=4)$ volume ratio of 3:1:2 as extraction agent, to determine the antibiotic in sludge sediments ,and the recovery rate was $57.4 \%-104.6 \%$. In view of the research conducted so far, the acetonitrile and water were used as an extraction agent, the extraction conditions were optimized by orthogonal test, so as to improve the recovery rates of sulfadiazine and sulfamethazine.

\section{Materials and methods}

\section{Instruments and reagents}

Primary Instruments: ACQUITF high performance liquid chromatograph, QSE-12 Solid phase extraction apparatus (Shanghai Jingxin Corporation), Thermostatic oscillator, DN-12A Nitrogen blowing instrument (Wuxi Jiuping Instrument Corporation), analytical balance (Ohaus Corporation Corporation), Thermo high-speed centrifuge (Thermo Fisher Corporation), ultrasonic cleaner (Shanghai Branch ultrasonic instrument limited Corporation), freeze dryer, HLB Solid phase extraction column (6ml/200mg, Shanghai Amp Corporation), $\mathrm{NH}_{2}$ purification column $(6 \mathrm{ml} / 200 \mathrm{mg}$, Shanghai Amp Corporation).

Reagent: Methanol (HPLC grade, Merck Chemical Technology Corporation), acetonitrile (HPLC grade, Merck Chemical Technology Corporation), Acetone (HPLC, Sinopharm Chemical Reagent Co., Ltd.), Formic acid (purity 98\%, Sinopharm Chemical Reagent Co., Ltd.), Ammonia (GR, Sinopharm Chemical Reagent Co., Ltd.), Hydrochloric acid (GR, Sinopharm Chemical Reagent Co., Ltd.), Ethylenediamine tetra-acetic acid disodium (EDTA-2Na, AR, Beijing Chemical Corporation), Deionized water is used in laboratory. Sulfadiazine (Beijing Bailingwei) purity 99\%, Sulfamethazine (TCI Chemical Industrial Corporation) purity of more than $98 \%$.

\section{Standard solution preparation}

Methanol was used as a solvent to prepare the standard storage solution with a mass concentration of $100 \mathrm{mg} / \mathrm{L}$, which was stored at $4{ }^{\circ} \mathrm{C}$, and the standard solution was diluted with deionized water to prepare standard solution

\section{Sample collection}

The sludge for experiment was collected from the city of Shanghai Songjiang District sewage treatment plant sludge by plate filter press after $(81.56 \%$ moisture content). Sludge samples collected were wrapped with aluminum foil and were immediately refrigerated the laboratory and stored at -20 ${ }^{\circ} \mathrm{C}$. After 12 hours freeze drying for 12 hours they were dry grinded in a mortar. Then the samples were 100 mesh sieved, after that they were mixed evenly and transferred to a closed glass bottle at 4 ${ }^{\circ} \mathrm{C}$ for preservation.

\section{HPLC conditions}

Chromatographic column: Athena $\mathrm{C}_{18}$ column (4.6 x 150mm, 5um, Shanghai Amp Corporation), column temperature: $40^{\circ} \mathrm{C}$, sample temperature: $4{ }^{\circ} \mathrm{C}$, sample volume: $20 \mu 1$, flow rate: $1 \mathrm{ml} / \mathrm{min}$.

Mobile phase: A was methanol, B was $0.1 \%$ formic acid aqueous solution; mobile phase ratio: methanol was $30 \%$, the aqueous solution of $0.1 \%$ formic acid was $70 \%$.

UV detection wavelength: with reference to the relevant literature, sulfonamides detection wavelength at was $260-275 \mathrm{~nm}$. A considerable part of the selection of the detectied wavelength was $265 \mathrm{~nm}{ }^{[12]}$. Through the experiments of two sulfonamides standard samples in the $265 \mathrm{~nm}$ wavelength response was strong, sensitivity was high. Therefore, in this article the actual detection wavelength was at $265 \mathrm{~nm}$. 


\section{Sample pretreatment}

\section{Sample extraction}

1.00 gram of sludge was added to $50 \mathrm{ml}$ centrifuge tube. Then added $10 \mathrm{ml}$ of acetonitrile water (1:3) solution with ammonia and adjusted $\mathrm{pH}$ to about 10.0, the solution was shaken for $20 \mathrm{~min}$ at 35 $\mathrm{Hz}$ ultrasonic extraction $15 \mathrm{~min}$, centrifugal $15 \mathrm{~min}(12000 \mathrm{r} / \mathrm{min})$. After the remove of the supernatant and residue add $10 \mathrm{ml}$ of extraction solution then the above operation was repeated twice. The extraction liquid was diluted with deionized water to $200 \mathrm{ml}$.

\section{Sample purification and concentration}

$0.3 \mathrm{~g}$ of EDTA-2Na was added to the dilution liquid, $\mathrm{pH}$ value was adjusted with hydrochloric acid to 3.0. The dilution of all HLB cartridges (activation: $6 \mathrm{ml}$ methanol, $6 \mathrm{ml} 5 \% \mathrm{pH} 3 \mathrm{EDTA}-2 \mathrm{Na}$ aqueous solution), when all the diluted sample completion, the $10 \mathrm{ml}$ of water was used to leach and vacuum. Then, $10 \mathrm{ml}$ methanol was used to elute. The eluent flowed through $\mathrm{NH}_{2}$ cartridge (activation: $6 \mathrm{ml}$ acetone), then use $6 \mathrm{ml}$ acetone - methanol - formic acid (500:500:1) solution and 6 $\mathrm{ml}$ acetone - formic acid (1000:1) solution was used for elution. The eluate was in soft under a stream of nitrogen blowing, blowing off to $1 \mathrm{ml}$, then used $10 \%$ methanol water solution to the sample bottle, volume $1 \mathrm{ml}$, sealed refrigeration in $4^{\circ} \mathrm{C}$ to be saved, high performance liquid chromatography on the determination.

\section{Results and analysis}

\section{Standard curve drawing}

Standard dilution liquid reserves, with a standard solution of certain concentration. Using $50 \mu 1$ micro syringe, volume of each sample was $20 \mu \mathrm{l}$, the concentration of $0.1 \mathrm{mg} / \mathrm{L}, 0.2 \mathrm{mg} / \mathrm{L}, 0.5 \mathrm{mg} / \mathrm{L}$, $1.0 \mathrm{mg} / \mathrm{L}, 2.0 \mathrm{mg} / \mathrm{L}, 5.0 \mathrm{mg} / \mathrm{L}, 10 \mathrm{mg} / \mathrm{L}, 100 \mathrm{mg} / \mathrm{L}$ of standard solution was determined by HPLC respectively. The standard curve was drawn using these values, the correlation coefficients were found to be greater than 0.99 .

According to the retention time of sulfonamide antibiotics, compounds to be measured were identified. For the standard curves of 2 sulfonamide antibiotics (Table 1). The recovery rates for the two target antibiotics were $75.50 \%, 72.79 \%$ respectively, the correlation coefficient of two standard curves was $99.89 \%$. Thus, this method possesses a good linear range and coefficient, and thus can satisfy the quantitative analysis needed for sulfonamide antibiotics.

Table 1 Retention time, linear relationship and recovery rate of two sulfonamides

\begin{tabular}{|c|c|c|c|c|}
\hline Sulfonamides & $\begin{array}{l}\text { Retention } \\
\text { time ( } \min )\end{array}$ & $\begin{array}{l}\text { Linear equation } \\
\text { of standard curve }\end{array}$ & $\begin{array}{l}\text { Correlation } \\
\text { coefficient }\left(\mathrm{R}^{2}\right)\end{array}$ & $\begin{array}{l}\text { Recovery } \\
\text { rate }(\%)\end{array}$ \\
\hline $\begin{array}{l}\text { Sulfadiazine } \\
\text { (SDZ) }\end{array}$ & 2.959 & $\begin{array}{l}y=1.5715 x-0.094 \\
6\end{array}$ & 0.9989 & 75.50 \\
\hline $\begin{array}{l}\text { Sulfamethazine } \\
\text { (SDMD) }\end{array}$ & 5.201 & $\begin{array}{l}y=1.3172 x-0.055 \\
5\end{array}$ & 0.9989 & 72.79 \\
\hline
\end{tabular}




\section{Single factor test}

\section{Study on the concentration of extraction agent}

Acetonitrile in different concentrations (acetonitrile concentration were 15\%, 25\%, 50\%, 75\%) as the extraction solvent, ultrasonic and other operations under the 2.1 extraction methods, different concentration of extraction agent of sulfonamide antibiotics recovery effect is shown in Figure 1.

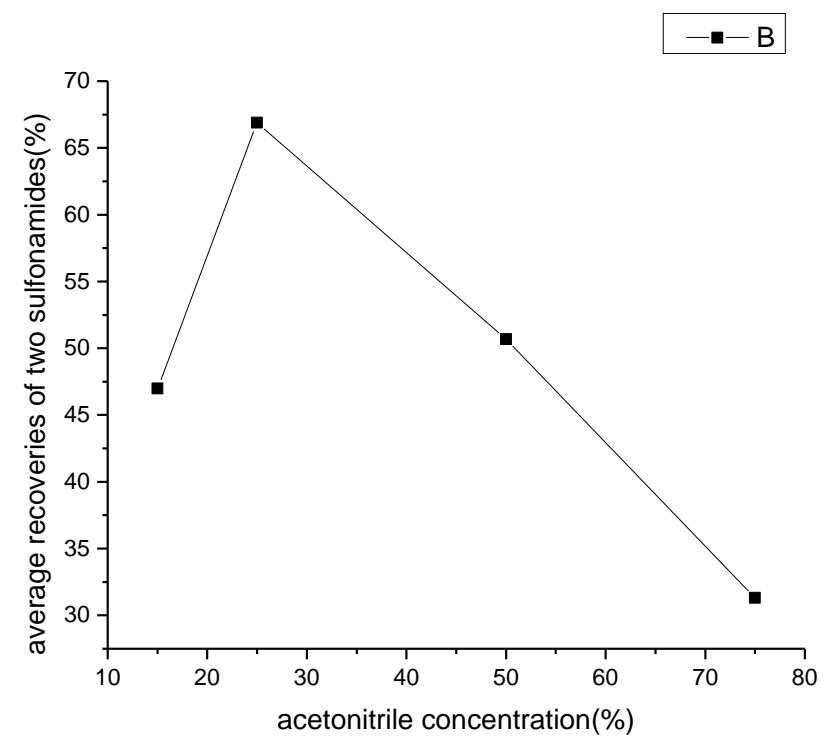

Figure 1 Effects of different acetonitrile concentration on the recovery of two sulfonamides

Figure 1 shows that different concentrations of acetonitrile as the extraction solvent, with the increase of the concentration of acetonitrile, the average extraction content of sulfonamide antibiotics firstly increases and then decreases. When the concentration of acetonitrile was $25 \%$ (acetonitrile: water $=1: 3$ ), the extraction efficiency goes to the highest. Therefore the ideal range was found to be $25 \%$ of acetonitrile as an extraction solvent.

\section{Investigation of oscillation time}

A acetonitrile with a concentration of $25 \%$ was chosen as the solvent for the process. The time of oscillation was investigated at 10,15, 20 and $25 \mathrm{~min}$. The other operations were treated with 2.1 ultrasonic extraction methods. The effect of different shaking time on the recovery rate of sulfonamide antibiotics was shown in Figure 2.

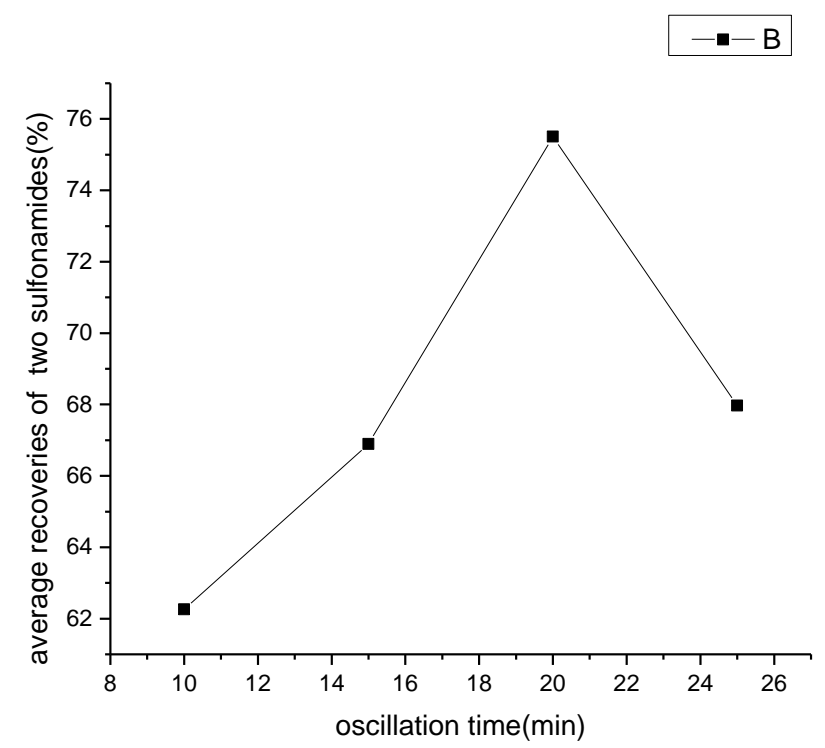

Figure 2 Effects of different oscillation time on the recovery of two sulfonamides 
Figure 2 shows that with the increase of oscillation time, the average content of sulfonamide antibiotics showed a decreasing trend after the first increase, when the oscillation time was 20 min. The average content of sulfonamide antibiotics reaches to the highest point at, so $20 \mathrm{~min}$ therefore it is the ideal time for ultra-sonication.

\section{Investigation of ultrasonic frequency}

$25 \%$ acetonitrile was chosen as an extraction solvent, the ultrasonic frequency of 35, 40 and 53 $\mathrm{Hz}$ was examined and the others operations were treated with ultrasonic extraction methods. The effects of different ultrasonic frequencies on the recovery rates of sulfonamides were compared with the results of Figure 3.

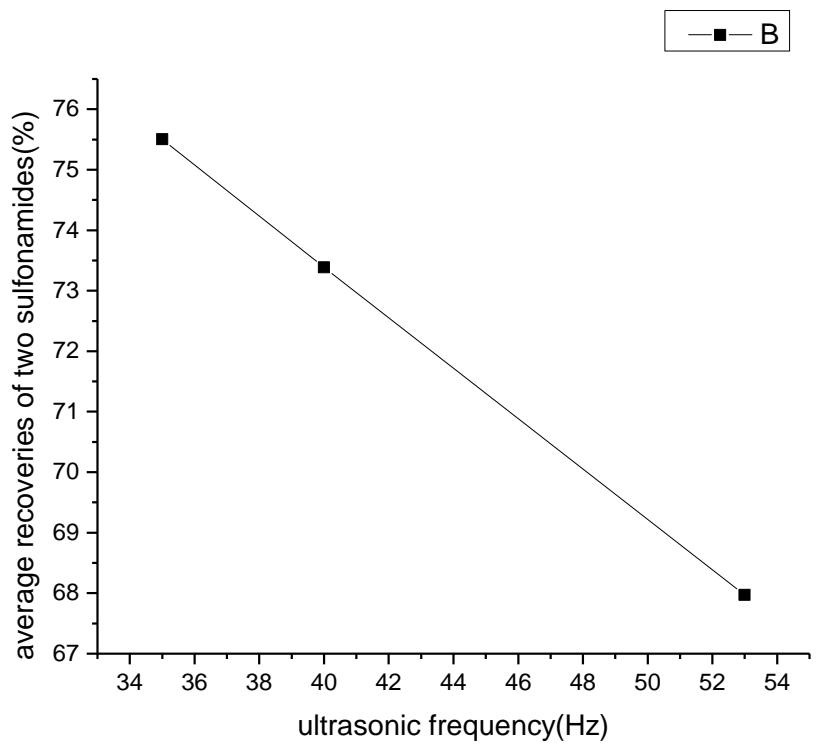

Figure 3 Effects of different ultrasonic frequency on the recovery of two sulfonamides

Figure 3 shows that with the increase of ultrasonic frequency, the average content of sulfonamide antibiotics showed a decreasing trend, when the ultrasonic frequency was $35 \mathrm{~Hz}$, the average content of sulfonamide antibiotics reaches its highest, therefore $35 \mathrm{~Hz}$ is the ideal ultrasonic frequency.

\section{Investigation of ultrasonic time}

$25 \%$ acetonitrile was used as an extraction solvent. The ultrasonic time was examined at 10, 15, 20 and $25 \mathrm{~min}$, and the other operations were treated with 2.1 ultrasonic extraction methods. The effects of different ultrasonic time on the recovery rates of sulfonamide antibiotics were shown in Figure 4. 


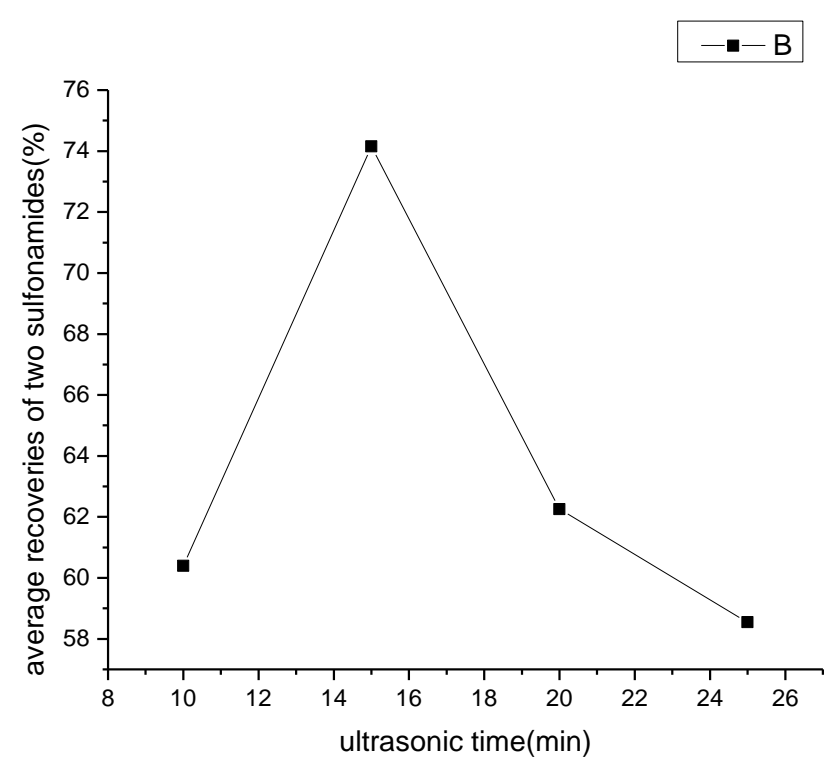

Figure 4 Effects of different ultrasonic time on the recovery of two sulfonamides

Figure 4 shows that with the increase of ultrasonic time, the average content of sulfonamide antibiotics showed a decreasing trend after the first increase, when the ultrasonic time was 15 min, the average content of sulfonamide antibiotics reaches the highest point, therefore $15 \mathrm{~min}$ is the ideal for ultra-sonication time.

\section{Orthogonal test}

Selection of extraction agent concentration (acetonitrile: water) (A), oscillation time (B), ultrasonic frequency $(\mathrm{C})$ and ultrasonic time (D) are the four factors under consideration to conduct three level test. In which the extraction agent concentration were 1:1,1:3,3:1, the corresponding number of 1 3 (below), the oscillation time selection was for 10, 15 and $20 \mathrm{~min}$, the ultrasonic frequency selection was $35,40,53 \mathrm{~Hz}$, the ultrasonic time selection was 10,15 and $20 \mathrm{~min}$. In this orthogonal experiment, it can be seen that in $\mathrm{A}_{2} \mathrm{~B}_{3} \mathrm{C}_{1} \mathrm{D}_{2}$ group (the extraction agent concentration was 1: 3, the oscillation time was $20 \mathrm{~min}$, ultrasonic frequency was $35 \mathrm{~Hz}$, ultrasonic time was $15 \mathrm{~min}$ ), the average recovery rates of sulfonamide antibiotics were $74.15 \%$, and the results were consistent with the previous single factor experiments.

Table 2 Orthogonal experiment table

\begin{tabular}{cccccc}
\hline & \multicolumn{3}{c}{ Influence factors } & & $\begin{array}{c}\text { Average recoveries of } \\
\text { two sulfonamides (\%) }\end{array}$ \\
\cline { 2 - 4 } Project & $\mathrm{A}$ & $\mathrm{B}$ & $\mathrm{C}$ & $\mathrm{D}$ & 46.31 \\
\hline $\mathrm{A}_{1} \mathrm{~B}_{1} \mathrm{C}_{1} \mathrm{D}_{1}$ & 1 & 1 & 1 & 1 & 46.99 \\
$\mathrm{~A}_{1} \mathrm{~B}_{2} \mathrm{C}_{2} \mathrm{D}_{2}$ & 1 & 2 & 2 & 2 & 50.68 \\
$\mathrm{~A}_{1} \mathrm{~B}_{3} \mathrm{C}_{3} \mathrm{D}_{3}$ & 1 & 3 & 3 & 3 & 62.26 \\
$\mathrm{~A}_{2} \mathrm{~B}_{1} \mathrm{C}_{2} \mathrm{D}_{3}$ & 2 & 1 & 2 & 3 & 66.89 \\
$\mathrm{~A}_{2} \mathrm{~B}_{2} \mathrm{C}_{3} \mathrm{D}_{1}$ & 2 & 2 & 3 & 1 & 74.15 \\
$\mathrm{~A}_{2} \mathrm{~B}_{3} \mathrm{C}_{1} \mathrm{D}_{2}$ & 2 & 3 & 1 & 2 & 31.32 \\
$\mathrm{~A}_{3} \mathrm{~B}_{1} \mathrm{C}_{3} \mathrm{D}_{1}$ & 3 & 1 & 3 & 1 & 26.94 \\
$\mathrm{~A}_{3} \mathrm{~B}_{2} \mathrm{C}_{1} \mathrm{D}_{3}$ & 3 & 2 & 1 & 3 & 27.68 \\
$\mathrm{~A}_{3} \mathrm{~B}_{3} \mathrm{C}_{2} \mathrm{D}_{2}$ & 3 & 3 & 2 & 2 & \\
\hline
\end{tabular}


In the data analysis of orthogonal experiment, the mean concentration of the extraction of three level tests were 47.993, 67.767 and 28.647 respectively, the range was 39.120 ; the mean shaking time of three levels were 46.630, 46.940 and 50.837, range was 4.207; the mean ultrasonic frequency of three levels were 49.133, 45.643 and 49.630, range was 3.987; the mean ultrasonic time of three levels were 48.173, 49.607 and 46.627, range was 2.980. From range comparison of four indicators, the changes in extraction agent concentrations had the most significant effect on the recovery rates of sulfonamide antibiotics, followed by the shock time, while the least impact was the ultrasonic time. Considering the four factors, take levels relative to the largest value for relative optimum namely $\mathrm{A}_{2} \mathrm{~B}_{3} \mathrm{C}_{1} \mathrm{D}_{2}$. Under these conditions, the average recovery rate of sulfonamide antibiotics was $74.15 \%$, and the relative standard deviation (RSD) was $9.085 \%$.

\section{Conclusions}

(1)The method of ultrasonic solid phase extraction was used to optimize the extraction method of sulfonamide antibiotics and the aim of improving the recovery rate was achieved.

(2) To determine the factors of the orthogonal experiment by single factor test, namely the selection of extraction agent concentration (acetonitrile: water), oscillation time, ultrasonic frequency and ultrasonic time as four factors of orthogonal experiment. The extraction agent concentration of 1:3 was determined by orthogonal experiment, the oscillation time was $20 \mathrm{~min}$, the ultrasonic frequency was $35 \mathrm{~Hz}$, ultrasonic time was $15 \mathrm{~min}$. Under these conditions, the recoveries of 2 sulfonamides were $75.50 \%$ and $72.79 \%$ respectively.

(3) It is a specific target, which can be used for the determination of many kinds of sulfonamide antibiotics in sludge simultaneously, and can help to conduct the trace analysis of pollutants in sludge.

\section{Acknowledgements}

This work was financially supported by "Textile Light" Application Basic Research fund in China (J201503) and Steel joint funds by National Natural Science Foundation in China-China's bao wu steel co., LTD (U1660107).

\section{References}

[1] D.W. Kolpin, E.T. Furlong, M.T. Meyer, et al. Environ Sci Technol, 36(6): 1202-1211(2002).

[2] A. Göbel, C.S. McArdell, A. Joss, et al. Sci Total Environ, 372(2): 361-371(2007).

[3] Jinhua He, Jinrong Qiu, Dechun He, et al. Guangdong Agricultural Sciences, 39(7): 225-229(2012). (In Chinese)

[4] Na Wang. Nanjing University, (2014). (In Chinese)

[5] O.A. Jones, J.N. Lester, N. Voulvoulis. Trends Biotechnology, 23: 163-167(2005).

[6] Caiming Tang, Qiuxin Huang, Yiyi Yu, Xianzhi Peng. Chinese Journal Analytical Chemistry, 37(8): 1119-1124(2009).

[7] Feng Chen, Guangguo Ying, Jifeng Yang, et al. Journal of Environmental Science and Health, Part B, 45(7): 682-693(2010).

[8] Mingyue Li, Qian Sun, Qin Wang, et al. Journal of Environmental Engineering, 10(2): 985-991(2016). (In Chinese)

[9] Shuo Wang, Jing Zhang, Bing Shao. Journal of Instrumental Analysis, 32(2): 179-185(2013). (In Chinese)

[10] Xiaoxia Jiang. Shenzhen University, (20150. (In Chinese)

[11] Jiali Ding, Yue Liu, Wei Zheng, et al. environmental science, 10: 3918-3925(2015). (In Chinese)

[12] Ping Wang, Jie Li, Hehui Zheng. Chromatographic, 25(5): 743-746(2007). (In Chinese) 